Original Article

\title{
The effect of neuromuscular electrical stimulation on functional status and quality of life after knee arthroplasty: a randomized controlled study
}

\author{
Demet Tekdos Demircioglu, MD ${ }^{1)^{*}}$, Nurdan Paker, MD²), Elif Erbil, MD, PhD²), \\ Derya Bugdayci, MD ${ }^{2)}$, Tuluhan Yunus Emre, MD ${ }^{3)}$ \\ 1) Department of Physical Medicine and Rehabilitation, Memorial Hizmet Hospital: Istanbul, Turkey \\ 2) Department of Physical Medicine and Rehabilitation, Istanbul Physical Medicine and Rehabilitation, \\ Educational and Research Hospital, Turkey \\ 3) Department of Orthopaedia and Traumatology, Memorial Hizmet Hospital, Turkey
}

\begin{abstract}
Purpose] The aim of this study was to investigate the effect of the addition of NMES to the post-TKA rehabilitation protocol on the functional status and quality of life of the patients. [Subjects and Methods] Patients were randomized into an exercise (control) and electrical stimulation (NMES) group. A home exercise program was prescribed for the control group. For the neuromuscular stimulation group 30 minute electrical stimulation applied to the vastus medialis muscle 5 days a week for 4 to 6 weeks. VAS, the timed up and go test, WOMAC and SF-36 scores were evaluated preoperatively and postoperatively at the first month and the third month of the follow-up period. [Results] Both the NMES group had 30 patients each, with 2 and 1 male patients respectively. The comparisons of WOMAC results at month 1 revealed that pain, stiffness, and total scores of the NMES group was significantly better than those of control group at the first and third months. Significantly better physical function and SF-36 subscales, except mental health, were found for the NMES group at the first month of follow-up. [Conclusion] The inclusion of the neuromuscular electrical stimulation program after knee arthroplasty was more effective at providing rapid improvements in knee pain, walking distance and quality of life.

Key words: Quadriceps muscle, Knee joint, Arthroplasty
\end{abstract}

(This article was submitted Mar. 23, 2015, and was accepted May 5, 2015)

\section{INTRODUCTION}

Total knee arthroplasty (TKA) is a surgical treatment which is useful in knee osteoarthritis (OA) for relieving pain and improving function ${ }^{1)}$. Even though TKA provides significant pain relief and improves self reported function, this procedure fails to improve the quadriceps muscle strength to the normal level for age-matched healthy population ${ }^{2)}$. Patients with endstage knee osteoarthritis exhibit relatively $20 \%$ more activation deficits in the involved quadriceps than in the uninvolved limb suggesting that neural mechanisms contribute to the weakness of the quadriceps even prior to TKA surgery ${ }^{3-6)}$.

Weakness of the quadriceps muscle results in important functional consequences such as decreased gait speed, balance, difficulty with stair climbing, and rising from a chair and risk of falls ${ }^{7)}$. Individuals with tibiofemoral osteoarthritis demonstrate significant quadriceps weakness compared

*Corresponding author. Demet Tekdos Demircioglu (E-mail: drtekdos@yahoo.com)

C2015 The Society of Physical Therapy Science. Published by IPEC Inc. This is an open-access article distributed under the terms of the Creative Commons Attribution Non-Commercial No Derivatives (by-ncnd) License $<$ http://creativecommons.org/licenses/by-nc-nd/3.0/>. to people without radiological evidence of the disease.

Pre- and post-operative strength loss is another issue which may result in long-term quadriceps strength loss ${ }^{8)}$. Three to four weeks after TKA surgery, patients experience $60 \%$ decrease in quadriceps strength and both pre- and post-operative loss of strength may be attributed to impaired quadriceps activation. However central activation deficits are reported to account for two times greater strength loss than muscle atrophy early after surgery ${ }^{3)}$.

Neuromuscular electrical stimulation (NMES) is often used as a quadriceps strengthening modality to train patients without sufficient volitional quadriceps activation by engaging neurophysiological mechanisms which are believed to facilitate strength gains and provide general physical stress to quadriceps neuromuscular system of the quadriceps ${ }^{9)}$.

NMES may affect central activation deficits, thereby allowing restoration of normal quadriceps muscle function more effectively than voluntary exercise alone. The improvement mechanism of NMES is unclear, however there are some theories. First the intensity of the muscle contraction with NMES may be greater ${ }^{10)}$. Second, NMES may alter motor recruitment. Electrically elicited muscle contractions enable activation of a greater proportion of type 2 muscle fibers than volitional exercise of comparable intensity. Finally NMES may also influence motor performance via peripheral afferent inputs that may alter motor cortex excitability ${ }^{11)}$. 
Various rehabilitation programs are available for strengthening the quadriceps of the patients with knee osteoarthtiris before or after the TKA procedure. The exercise programs include isometric exercises, simple strengthening exercises, gait training and closed kinetic chain exercises to improve strength and functional recovery $9,12,13$ ).

There are conflicting evidence in terms of the effectiveness of NMES in improving quadriceps strength and physical performance of patients following TKA. The settings, application methods, overall intensity, duration and postoperative timing differ substantially among studies. The results of previous studies are controversial as NMES has been found effective in improving physical performance in two studies ${ }^{14)}$, ineffective in another study compared to exercise alone $^{15)}$ and another study (designed as a non-inferiority trial) reporting that the isolated use of NMES is as effective as traditional physical therapy following $\mathrm{TKA}^{16)}$.

According to a review, home exercise programs consisting of different exercise protocols provide short term improvements in the physical functioning of post-TKA patients, but these improvements persist for less than one year, and different exercise programs have no superiority over each other ${ }^{17)}$. Valid and acceptable scales are required to analyze the outcomes of treatments for osteoarthritis patients ${ }^{18}$.

The primary aim of this study was to investigate the effect of the addition of NMES to the post-TKA rehabilitation protocol on patients' functional status and quality of life. Secondary aim was the assessment of the changes in patients' pain and range of motion. Our hypothesis was that the supplementation of exercises with NMES would yield further improvement in patients' functions and quality of life at 3 months after TKA.

\section{SUBJECTS AND METHODS}

Sixty patients with knee OA who were admitted to the Orthopaedia and Traumatology outpatient clinic for TKA of the Istanbul Physical Medicine and Rehabilitation Training Hospital, Istanbul, Turkey between 01-September-2006 and 01-April-2007 were included in this study.

All patients received a physical examination, and knee roentgenograms were taken. The patients who had symptomatic hip osteoarthritis, concomitant cardiac or internal diseases precluding surgical treatment, a history of epilepsy, the presence of a pacemaker, a skin lesion over the quadriceps muscle that required electrode application, muscle atrophy, or severe cognitive dysfunction were excluded from the study.

Patients were hospitalized in the department of orthopedics inpatient clinic and a total knee arthroplasty procedure was scheduled. Demographic characteristics of the patients were obtained from paients' records. Passive knee range of motion (ROM) was measured in the supine position using a manual goniometer. Passive knee ROMs were measured and recorded. The Timed up and Go test (TUG), and Western Ontario McMaster Osteoarthritis Index (WOMAC) were conducted for the assessment of knee functions and symptoms before surgery, and Short-Form 36 (SF-36) for the determination of their general health status.

The patients were randomized into two groups by flip- ping a coin. The head side of the coin determined the control group, and the tail side of the coin determined NMES group. Beginning from the postoperative day 1, patients in both groups started 30 minute sessions of range of motion (ROM) exercises from 0 to $30^{\circ}$ within the limits of pain, using a continuous passive motion (CPM) device, for one week. They also attended a rehabilitation program beginning from the postoperative first day. Ankle ROM exercises were started on postoperative day 0 . Patients were taught to perform isometric quadriceps exercises and to raise their leg straight, stand up with a walker and fully extend their knees and active and assisted ROM exercises were started on the first postoperative day. Active ROM and isometric quadriceps exercises were continued between postoperative days 2 and 10. After the removal of the sutures, patellar mobilization was performed for patients who did not develop postoperative problems. Patients were instructed to perform active hip abduction and adduction exercises. A home exercise program was recommended and patients were discharged from the hospital on postoperative day 7 . Walkerassisted ambulation was recommended until the sixth week after surgery. Closed kinetic chain exercises were started from 4 to 5 weeks after surgery. CPM applications and a rehabilitation program were also started in NMES group on the first day after surgery. In this group, 30-minute sessions of NMES were applied to the vastus medialis muscle 5 days a week, for 4 to 6 weeks.

Following the surgery, a bandage was applied to the knee and limb elevation was used for all patients. Cryotherapy was conducted for 15 minutes, three times a day to reduce pain and non-opioid analgesics were administered to the patients with severe pain.

A portable stimulator was used for the NMES intervention (Compex ${ }^{\circledR}$ DJO company). During the NMES treatment, the lower extremity was secured with self adhesive straps to a stable chair to allow approximately 85 degrees of hip flexion and 60 degrees of knee flexion. Large rectangular electrodes were used to maximize treatment tolerance and effectiveness. Active electrodes were placed over the vastus medialis muscle. The negative pole of the channel was placed longitudinally in a position parallel and distal to the active electrode. Stimuli were given to provide powerful muscle contractions without pain. The current intensity was adjusted in the range from $28 \mathrm{~mA}$ to $90 \mathrm{~mA}$, according to subjects' tolerance. Training duration was 30 minutes and the frequency varied between 30 and $100 \mathrm{~Hz}$. The contraction time, rest time, ramp up time, and ramp down time were respectively $10,10,1.5$ and $0.75 \mathrm{~s}$. Biphasic, symmetrical synchronized, sequential $400 \mu$ s pulse-width waves were used.

The follow up visits were scheduled for the first month and third month after surgery. Knee ROMs were measured and TUG, WOMAC and SF-36 forms were completed during the follow up visits. All of the participants completed the study. Written consent to participation was obtained from each patient and approval for the study was obtained from the Ethics Committee of the hospital.

The WOMAC is a validated health questionnaire which is specific for hip and knee OA, and it consists of 24 questions scored from zero to four (best to worst respectively). These 
are subcategorized into pain (0-20), stiffness $(0-8)$ and function $(0-68)^{19)}$. The Turkish version has been validated ${ }^{20)}$.

SF-36 evaluates general health using a 36-item questionnaire. Component scores are given for physical and mental health. Both are scored from zero to 100 with a higher score indicating better health. The reliability and validity of the SF-36 are well established ${ }^{21)}$. The Turkish version was validated $^{22)}$.

TUG was used for the assessment of functionality and mobility. It measures the time (in seconds) required to stand up from a chair, walk 3 meters turn and return and sit on the chair. The participants wore comfortable shoes during the test. The average of 3 attempts was calculated ${ }^{23)}$.

The data were statistically analyzed using SPSS 11.5 for Windows. Descriptive statistics were preferred in order to analyze and classify the demographic and clinical characteristics of the patients. Student's t-test was used to analyze the parametric measurements of the exercise and NMES groups. Bivariate variance analysis was used for the intragroup analyses of the data obtained during the preoperative assessment and the follow-up visits at month 1 and month 3. Pearson's correlation coefficient was used to analyze non-parametric data. $\mathrm{P}$ values $<0.05$ were considered statistically significant.

\section{RESULTS}

Thirty ( 29 female) patients were included in the exercise (control) group and 30 (28 female) patients were included in the NMES group. The demographic data of the patients are summarized in the Table 1.

There was no significant difference between the NMES and control groups in terms of pre-operative VAS scores, knee flexion or extension, TUG scores or WOMAC scores $(p>0.05)$. In the NMES group, VAS scores in both the first and third months after TKA were found to be significantly better than in the control group $(\mathrm{p}=0.0)$. Knee flexion and extension ranges were significantly better in the NMES group than in the control group at the first-month follow-up visit $(p=0.05)$. Although the flexion and extension measurements were also better in the NMES group than those of the control group at the third-month follow up visit the difference between the two groups was not statistically significant.

While TUG test scores were significantly better in the NMES group at the first-month follow up visit $(p=0.04)$, no statistically significant difference was found between the groups in terms of TUG scores at the third-month follow up visit $(\mathrm{p}>0.05)$ (Table 2).

The comparisons of WOMAC results at Month 1 revealed that pain, stiffness, physical function and total scores of the NMES group was significantly better than those of control group $(\mathrm{p}=0.00)$. At Month 3, WOMAC pain, stiffness and total scores of the NMES group were significantly better than those of the control group, while no significant difference was found between the two groups in the WOMAC physical function subscores $(\mathrm{p}=0.02)$.

Preoperative SF-36 summary scores revealed that the mental component and total scores of SF-36 were higher in the control group, no significant difference was found between the two groups in physical component scores $(p=0.0)$.

SF-36 summary scores at the first postoperative month showed significantly better mental component, physical component and total scores in the NMES group, than in the control group $(\mathrm{p}=0.01)$. In the analyses of SF-36 summary scores at Month 3 the physical health, mental health and total scores of the NMES group patients were found to be significantly better than those of the control group $(\mathrm{p}=0.004)$ (Table 3).

Patients did not develop any complication other than postoperative pain. In the NMES group, a 1st degree skin burn developed in two patients during the electrical stimulation of the skin. For this reason, the electrode sites were changed and the sessions were continued. There were no significant difference between the NMES and control groups in age,

Table 1. Demographic characteristics of the groups

\begin{tabular}{lcccc}
\hline & \multicolumn{2}{c}{ Exercise group $(\mathrm{n}=30)$} & \multicolumn{2}{c}{ NMES group $(\mathrm{n}=30)$} \\
\hline & Means \pm SD & $\begin{array}{c}\text { Upper- } \\
\text { lower } \\
\text { limits }\end{array}$ & $\begin{array}{c}\text { Means } \\
\pm \mathrm{SD}\end{array}$ & $\begin{array}{c}\text { Upper } \\
\text { and lower } \\
\text { limits }\end{array}$ \\
\hline Age (years) & $64.6 \pm 6.6$ & $71-58$ & $66.2 \pm 7.2$ & $73-59$ \\
Height $(\mathrm{cm})$ & $159.9 \pm 6.9$ & $167-153$ & $158.4 \pm 4.9$ & $163-153$ \\
Weight $(\mathrm{kg})$ & $76.7 \pm 11.5$ & $88-65$ & $73.0 \pm 10.1$ & $83-63$ \\
BMI $\left(\mathrm{kg} / \mathrm{m}^{2}\right)$ & $30.1 \pm 4.6$ & $35-26$ & $29.1 \pm 3.9$ & $33-25$ \\
\hline
\end{tabular}

NMES: Neuromuscular electrical stimulation, BMI: Body Mass Index. Descriptive statistics were used for statistical analysis. $\mathrm{p}<0.05$ was considered statistically significant.

Table 2. Comparison of the groups in terms of pain, functional status and knee range of motions

\begin{tabular}{|c|c|c|c|c|c|c|}
\hline & \multicolumn{3}{|c|}{$\begin{array}{c}\text { NMES group }(\mathrm{n}=30) \\
\text { Means } \pm \text { SD (Upper-lower limits) }\end{array}$} & \multicolumn{3}{|c|}{$\begin{array}{c}\text { Exercise group }(\mathrm{n}=30) \\
\text { Means } \pm \text { SD (Upper-lower limits) }\end{array}$} \\
\hline & Preoperative & Month 1 & Month 3 & Preoperative & Month 1 & Month 3 \\
\hline Knee flexion & $99.3 \pm 16.9$ & $109.5 \pm 8.4^{*}$ & $113.2 \pm 7.7$ & $101.8 \pm 9.9$ & $105.6 \pm 7.1$ & $110.5 \pm 7.9$ \\
\hline (Degree) & $(116-82)$ & $(118-101)$ & $(121-105)$ & $(112-92)$ & (113-98) & $(119-101)$ \\
\hline Knee extension & $-6.9 \pm 4.1$ & $-0.5 \pm 1.5^{*}$ & $-0.3 \pm 1.3$ & $-7.6 \pm 3.5$ & $-1.1 \pm 2.2$ & $-0.5 \pm 1.5$ \\
\hline (Degree) & $(-2.8--11)$ & $(-2.0-1.0)$ & $(-1.6-1.0)$ & $(-2.1--11)$ & $(1.1-3.3)$ & $(-2.0-1.0)$ \\
\hline VAS & $\begin{array}{c}8.1 \pm 0.6 \\
(8.7-7.5)\end{array}$ & $\begin{array}{l}4.3 \pm 0.7 * \\
(5.0-3.6)\end{array}$ & $\begin{array}{l}2.9 \pm 0.5^{*} \\
(3.5-2.0)\end{array}$ & $\begin{array}{c}8.4 \pm 0.6 \\
(9-7)\end{array}$ & $\begin{array}{c}5.3 \pm 0.6 \\
(6-4)\end{array}$ & $\begin{array}{c}3.5 \pm 0.6 \\
(4-3)\end{array}$ \\
\hline $\begin{array}{l}\text { TUG times } \\
\text { (Second) }\end{array}$ & $\begin{array}{l}20.3 \pm 2.7 \\
(23-17.5)\end{array}$ & $\begin{array}{c}12.8 \pm 1.9^{*} \\
(14-11)\end{array}$ & $\begin{array}{r}12.3 \pm 2.1 \\
(14-10)\end{array}$ & $\begin{array}{l}20.4 \pm 2.5 \\
(23-24)\end{array}$ & $\begin{array}{l}13.7 \pm 1.5 \\
(15-12)\end{array}$ & $\begin{array}{l}12.9 \pm 1.9 \\
(15-12)\end{array}$ \\
\hline
\end{tabular}

NMES: Neuromuscular electrical stimulation, TUG: Timed Up and Go test, VAS: Visual Analog Scale

The paired t-test was used for statistical analysis . ${ }^{*}<0.05$ was considered statistically significant. 
height, weight, or BMI ( $\mathrm{p}>0.05)$.

\section{DISCUSSION}

Knee flexion and extension, and the TUG time one month after TKA were significantly better in the NMES group. However, there were no significant differences between the groups in terms of knee ROMs and TUG times at Month 3 of the follow-up. The results show there were statistically significant improvements in knee-related pain, stiffness and functions in both groups at one month after TKA. Quality of life was significantly better in the NMES group, than in the control group at Month 1. Both the physical and mental subscores of QOL in the NMES group were found to be significantly better than those of the control group at Month 3 .

In a study the effectiveness of electrical stimulation in total knee arthroplasty, Avramidis et al. applied electrical stimulation to the vastus medialis during the first 6 weeks after surgery, along with a conventional rehabilitation program, but they did not find any significant improvements in quality of life at weeks 6 and 12, in comparison to the preoperative scores ${ }^{24)}$

Quadriceps atrophy of type 1 and type 2 muscle fibers occurs in age-related osteoarthritis and the atrophy of type 2 muscle fibers is particularly responsible for muscle weakness. NMES affects type 2 muscle fibers and the addition of NMES to the postoperative treatment of exercise programs, potentializes muscle strength enhancement. In a study conducted by Lewek et al., significant improvements in ROMs, muscle strength, and pain resolution were observed 12 sessions of electrical stimulation of the vastus medialis muscle after total knee arthroplasty ${ }^{25)}$.

Gotlin et al., used a conventional rehabilitation protocol and CPM for a control group of subjects and a conventional rehabilitation protocol, CPM and NMES for an experimen- tal groups of subjects to study the differences between the degrees of active and passive knee extension before surgery and the degrees of active and passive knee extension at discharge of 40 patients with TKA. They found the difference between passive and active extension was significantly lower in experimental group. The mechanism causing limited knee extension is not clearly understood. However, resection of the anterior cruciate ligaments during the knee arthroplasty procedure is considered to decrease the number of afferent receptors eventually resulting in limited knee extension. Furthermore, one suggested that this mechanism is associated with a reflex inhibitory response, and that earlier functional improvements might be obtained by NMES through the inhibition of this mechanism ${ }^{26}$ ). The degree of flexion at baseline is a significant predictor of the degree of postoperative knee flexion. Higher degrees of knee flexion at the baseline were associated with smaller ROM gains, while the ROM gain was greater in a previously stiff knee ${ }^{27)}$. Knee flexion ranges in the present study increased postoperatively. All patients registered increases in knee flexion except one patient who had a knee flexion loss. The improvements in knee flexion and extension ranges were significantly higher in the NMES group than in the control group at postoperative Month 1.

Mizner et al. assessed the effects of preoperative quadriceps muscle strength, ROM and pain on the functional state and quality of life at postoperative year 1 in a study of 40 TKA patients. At the end of the present study, the most important determinant of the quality of life and functional state were reported to be the quadriceps muscle strength ${ }^{28)}$. In another study 276 patients attending a post-TKA rehabilitation program were evaluated by Jones et al. before the surgery and at Month 6 postoperatively using WOMAC and SF-36. Significant improvements were observed in the post-TKA patients' WOMAC pain and function scores, and

Table 3 . Comparison of the WOMAC and SF-36 subcale scores of the groups.

\begin{tabular}{lcccccc}
\hline & \multicolumn{3}{c}{$\begin{array}{c}\text { NMES Group } \\
(\mathrm{n}=30)\end{array}$} & \multicolumn{3}{c}{$\begin{array}{c}\text { Exercise Group } \\
(\mathrm{n}=30)\end{array}$} \\
\hline & \multicolumn{3}{c}{ Means \pm SD (Upper-lower limits) } & \multicolumn{2}{c}{ Means \pm SD (Upper-lower limits) } \\
\hline Preoperative & Month 1 & Month 3 & Preoperative & Month 1 & Month 3 \\
WOMAC & $78.8 \pm 12.4$ & $61.8 \pm 10.5^{*}$ & $42.8 \pm 16.8^{*}$ & $90.7 \pm 8.9$ & $83.5 \pm 12.3$ & $48.5 \pm 14.2$ \\
WOMAC & $(91-66)$ & $(72-51)$ & $(60-26)$ & $(100-82)$ & $(96-71)$ & $(62-34)$ \\
stiffness & $85.8 \pm 13.8$ & $70.4 \pm 8.4^{*}$ & $42.9 \pm 12.6^{*}$ & $92.1 \pm 9.6$ & $76.7 \pm 11.2$ & $47.9 \pm 12.3$ \\
WOMAC & $(100-72)$ & $(79-62)$ & $(56-30)$ & $(100-83)$ & $(88-66)$ & $(60-36)$ \\
function & $80.5 \pm 4.9$ & $51.8 \pm 7.7^{*}$ & $44.5 \pm 12.5$ & $83.5 \pm 8.1$ & $77.1 \pm 11.0$ & $48.8 \pm 16.5$ \\
WOMAC & $(85-75)$ & $(60-44)$ & $(57-32)$ & $(92-75)$ & $(88-67)$ & $(66-32)$ \\
total & $78.5 \pm 4.8$ & $53.4 \pm 6.4^{*}$ & $42.3 \pm 11.3^{*}$ & $84.7 \pm 7.4$ & $77.3 \pm 9.5$ & $47.2 \pm 13.8$ \\
Sf-36 physical & $(83-73)$ & $(60-47)$ & $(54-31)$ & $(92-77)$ & $(87-68)$ & $(61-33)$ \\
health score & $36.1 \pm 21.7$ & $59.6 \pm 16.5^{*}$ & $68.5 \pm 14.8^{*}$ & $35.9 \pm 17.1$ & $44.4 \pm 21.5$ & $67.8 \pm 14.5$ \\
Sf-36 mental & $(58-14)$ & $(76-43)$ & $(83-54)$ & $(53-19)$ & $(66-23)$ & $(82-53)$ \\
health score & $44.8 \pm 16.2$ & $71.9 \pm 13.0^{*}$ & $65.1 \pm 12.1^{*}$ & $48.5 \pm 16.4^{*}$ & $58.6 \pm 15.9$ & $60.9 \pm 15.8$ \\
Sf-36 total & $(61-29)$ & $(85-59)$ & $(77-53)$ & $(65-32)$ & $(75-43)$ & $(77-45)$ \\
score & $39.9 \pm 19.4$ & $66.6 \pm 15.3^{*}$ & $68.0 \pm 11.6^{*}$ & $39.8 \pm 16.8^{*}$ & $50.7 \pm 17.3$ & $67.8 \pm 15.6$ \\
\hline
\end{tabular}

NMES: Neuromuscular electrical stimulation, WOMAC: Western Ontario McMaster Osteoarthritis Index

SF-36: Short Form 36. The Mann-Whitney U test was used for the statistical analysis.* $<0.05$ was considered statistically significant. 
in all the subscale scores of SF-36, except mental health and general health perception ${ }^{29}$ ). March et al. assessed 52 TKA patients preoperatively and post-operatively at month 12 , using the SF-36 form and found significant improvements, particularly in the physical functionality, pain, and physical role scores ${ }^{30)}$.

Parent et al., on the other hand, reported significant improvements in WOMAC physical function and SF physical functionality scores at postoperative Month 2, compared to preoperative scores in a study of 65 TKA patients ${ }^{31}$.

Shields et al. assessed the quality of life preoperatively and postoperative month 3 and month 6 using the SF-36 form and found that the greatest improvements were in the physical functionality, pain and vitality subscales. They reported that the scores of these scales improved in Month 3, that the improvements in limited physical role and emotional role occured in Month 6 postoperatively, and that these scores approached the scores of the reference population 3 to 6 months later. They suggested that the greatest improvement occurred within the first three months since the scores only slightly changed from the third month to the sixth month ${ }^{32}$. In the present study statistically significant improvements in knee related pain, stiffness and functions were found in both groups at Month 1 after TKA. Quality of life was significantly better in the NMES group, than in the control group at one month post-TKA. In the analyses of SF36 summary scores at 3rd month, the physical health, mental health and total scores of the NMES group were found to be significantly better than those of the control group.

Two patients in the present study experienced mild skin burns but they continued to participate in the study after relocation of the electrodes. A full-thickness skin burn reported as a result of electrical stimulation after unilateral knee arthroplasty in a previous study ${ }^{33)}$.

This study had certain strengths and limitations. The first strength of this study was the prospective randomized design, and the second was the use of the Turkish version of scales to evaluate the pain, function and quality of life. The third strength was all measurements were performed by same physician and without a long follow-up period. The limitations of this study were the relatively small number of the patients and statistical tests employed.

In conclusion, supplementation of the exercise program after TKA surgery was found to be more effective for the rapid improvement in knee functions and quality of life.

\section{REFERENCES}

1) American Academy of Orthopaedic Surgeons: Most commonly performed Musculoskeletal-Related Procedures. http://www.aaos.org/research /stats/ top-hospitalization_visits.pdf (Accessed July 24, 2011)

2) Hopkins JT, Ingersoll CD: Arthrogenic muscle inhibition: A limiting factor in joint rehabilitation. J Sport Rehabil, 2000, 9: 135-159.

3) Mizner RL, Petterson SC, Stevens JE, et al.: Early quadriceps strength loss after total knee arthroplasty. The contributions of muscle atrophy and failure of voluntary muscle activation. J Bone Joint Surg Am, 2005, 87: 1047-1053. [Medline] [CrossRef]

4) Morita S, Kusaka T, Tanaka S, et al.: The relationship between muscle weakness and activation of the cerebral cortex early after unicompartmental knee arthroplasty. J Phys Ther Sci, 2013, 25: 301-307. [CrossRef]

5) Petterson SC, Barrance P, Marmon AR, et al.: Time course of quad strength, area, and activation after knee arthroplasty and strength training. Med Sci Sports Exerc, 2011, 43: 225-231. [Medline] [CrossRef]
6) Stevens-Lapsley JE, Balter JE, Wolfe P, et al.: Early neuromuscular electrical stimulation to improve quadriceps muscle strength after total knee arthroplasty: a randomized controlled trial. Phys Ther, 2012, 92: 210-226. [Medline] [CrossRef]

7) Moreland JD, Richardson JA, Goldsmith $\mathrm{CH}$, et al.: Muscle weakness and falls in older adults: a systematic review and meta-analysis. J Am Geriatr Soc, 2004, 52: 1121-1129. [Medline] [CrossRef]

8) Palmieri-Smith RM, Thomas AC, Karvonen-Gutierrez C, et al.: Isometric quadriceps strength in women with mild, moderate, and severe knee osteoarthritis. Am J Phys Med Rehabil, 2010, 89: 541-548. [Medline] [CrossRef]

9) Kittelson AJ, Stackhouse SK, Stevens-Lapsley JE: Neuromuscular electrical stimulation after total joint arthroplasty: a critical review of recent controlled studies. Eur J Phys Rehabil Med, 2013, 49: 909-920. [Medline]

10) Snyder-Mackler L, Garrett M, Roberts M: A comparison of torque generating capabilities of three different electrical stimulating currents. J Orthop Sports Phys Ther, 1989, 10: 297-301. [Medline] [CrossRef]

11) Thomas AC, Stevens-Lapsley JE: Importance of attenuating quadriceps activation deficits after total knee arthroplasty. Exerc Sport Sci Rev, 2012, 40: 95-101. [Medline] [CrossRef]

12) Park SH, Hwangbo G: Effects of combined application of progressive resistance training and Russian electrical stimulation on quadriceps femoris muscle strength in elderly women with knee osteoarthritis. J Phys Ther Sci, 2015, 27: 729-731. [Medline] [CrossRef]

13) Anwer S, Alghadir A: Effect of isometric quadriceps exercise on muscle strength, pain, and function in patients with knee osteoarthritis: a randomized controlled study. J Phys Ther Sci, 2014, 26: 745-748. [Medline] [CrossRef]

14) Avramidis K, Karachalios T, Popotonasios K, et al.: Does electric stimulation of the vastus medialis muscle influence rehabilitation after total knee replacement? Orthopedics, 2011, 34: 175. [Medline]

15) Petterson SC, Mizner RL, Stevens JE, et al.: Improved function from progressive strengthening interventions after total knee arthroplasty: a randomized clinical trial with an imbedded prospective cohort. Arthritis Rheum, 2009, 61: 174-183. [Medline] [CrossRef]

16) Levine McElroy K, Stakich V, Cicco J: Comparing concentional physical therapy rehabilitation with neuromuscular electrical stimulation after TKA. Orthopedics, 2013, 36: 319-324. [CrossRef]

17) Minns Lowe CJ, Barker KL, Dewey ME, et al.: Effectiveness of physiotherapy exercise following hip arthroplasty for osteoarthritis: a systematic review of clinical trials. BMC Musculoskelet Disord, 2009, 10: 98. [Medline] [CrossRef]

18) Brazier JE, Harper R, Munro J, et al.: Generic and condition- spesific outcome measures for people with osteoarthritis of knee. Rheumatol, 1999, $870-877$.

19) Bellamy N, Buchanan WW, Goldsmith $\mathrm{CH}$, et al.: Validation study of WOMAC: a health status instrument for measuring clinically important patient relevant outcomes to antirheumatic drug therapy in patients with osteoarthritis of the hip or knee. J Rheumatol, 1988, 15: 1833-1840. [Medline]

20) Tüzün EH, Eker L, Aytar A, et al.: Acceptability, reliability, validity and responsiveness of the Turkish version of WOMAC osteoarthritis index. Osteoarthritis Cartilage, 2005, 13: 28-33. [Medline] [CrossRef]

21) McHorney CA, Ware JE Jr, Lu JF, et al.: The MOS 36-item Short-Form Health Survey (SF-36): III. Tests of data quality, scaling assumptions, and reliability across diverse patient groups. Med Care, 1994, 32: 40-66. [Medline] [CrossRef]

22) Kocyigit H, Aydemir O, Fisek G: Kisa Form-36 (KF-36)'nin Turkce versiyonun guvenirliligi ve gecerliligi (The reliability and validity of the Turkish version of SF-36). Ilac Tedavi Derg, 1999, 12: 102-106.

23) Podsiadlo D, Richardson S: The timed "Up \& Go": a test of basic functional mobility for frail elderly persons. J Am Geriatr Soc, 1991, 39: 142-148. [Medline] [CrossRef]

24) Avramidis K, Strike PW, Taylor PN, et al.: Effectiveness of electric stimulation of the vastus medialis muscle in the rehabilitation of patients after total knee arthroplasty. Arch Phys Med Rehabil, 2003, 84: 1850-1853. [Medline] [CrossRef]

25) Lewek M, Stevens J, Snyder-Mackler L: The use of electrical stimulation to increase quadriceps femoris muscle force in an elderly patient following a total knee arthroplasty. Phys Ther, 2001, 81: 1565-1571. [Medline]

26) Gotlin RS, Hershkowitz S, Juris PM, et al.: Electrical stimulation effect on extensor lag and length of hospital stay after total knee arthroplasty. Arch Phys Med Rehabil, 1994, 75: 957-959. [Medline]

27) Shakespeare D, Ledger M, Kinzel V: Flexion after total knee replacement. A comparison between the Medial Pivot knee and a posterior stabilised implant. Knee, 2006, 13: 371-373. [Medline] [CrossRef] 
28) Mizner RL, Petterson SC, Stevens JE, et al.: Preoperative quadriceps strength predicts functional ability one year after total knee arthroplasty. J Rheumatol, 2005, 32: 1533-1539. [Medline]

29) Jones CA, Voaklander DC, Johnston DW, et al.: Health related quality of life outcomes after total hip and knee arthroplasties in a community based population. J Rheumatol, 2000, 27: 1745-1752. [Medline]

30) March LM, Cross MJ, Lapsley H, et al.: Outcomes after hip or knee replacement surgery for osteoarthritis. A prospective cohort study comparing patients' quality of life before and after surgery with age-related population norms. Med J Aust, 1999, 171: 235-238. [Medline]
31) Parent E, Moffet H: Comparative responsiveness of locomotor tests and questionnaires used to follow early recovery after total knee arthroplasty. Arch Phys Med Rehabil, 2002, 83: 70-80. [Medline] [CrossRef]

32) Shields RK, Enloe LJ, Leo KC: Health related quality of life in patients with total hip or knee replacement. Arch Phys Med Rehabil, 1999, 80: 572-579. [Medline] [CrossRef]

33) Ford KS, Shrader MW, Smith J, et al.: Full-thickness burn formation after the use of electrical stimulation for rehabilitation of unicompartmental knee arthroplasty. J Arthroplasty, 2005, 20: 950-953. [Medline] [CrossRef] 